Hans P. Ogal

Die Neue Schädelakupunktur nach Yamamoto (YNSA - Yamamoto New Scalp Acupuncture) wurde in den 1960er-Jahren von Dr. med. Toshikatsu Yamamoto (Ph.D.) begründet. Er fand bei der Behandlung von Schmerz- und Lähmungspatienten mit klassischer Akupunktur und chinesischer Schädelakupunktur weitere schmerzhafte Punkte am Schädel, die somatoforme Zuordnungen des gesamten Körpers zeigen. Diese neue Akupunkturform hat er in den letzten 40 Jahren systematisiert und vervollständigt. Neben der Entdeckung der neuen Punkte am Kopf hat Yamamoto die orientalische Bauchdeckendiagnostik modifiziert und die auf ihr beruhenden Reflexzonen den Schädelakupunkturpunkten zugeordnet. Darüber hinaus fand er kongruent zur Bauchdeckendiagnostik und übereinstimmend mit den indizierten Punkten am Schädel eine weitere somatoforme Anordnung im Bereich des lateralen Halsdreiecks, das zur Diagnostik indizierter YNSAPunkte genutzt werden kann.

Auch weiterhin ist Yamamoto auf der Suche nach «neuen» Punkten zur schnellen und effektiven Behandlung unterschiedlichster Störungen. So hat er in den letzten Jahren Punkte für die 12 Hirnnerven gefunden und diese dem Meridiansystem zuordnen können.

Die YNSA ist geeignet zur schnellen und effizienten Behandlung von Schmerzsyndromen des Bewegungssystems, funktionellen Störungen innerer Organe und Lähmungen unterschiedlicher Genese. Der Behandlungserfolg bei der Schmerztherapie ist unmittelbar nach der korrekt applizierten Nadel zu erwarten.

\section{Neue Schädelakupunktur nach Yamamoto}

\section{Methodik/Anwendung}

Die zu behandelnden Punkte liegen an der Stirn-/Haar- bzw. Schläfen-/ Haargrenze. Die YNSA stellt im eigentlichen Sinne zwei Somatotope dar, die BASIS- und die YPSILONPunkte. Die BASIS-Punkte zeigen eine Unterteilung des Körpers im westlich-anatomischen Sinne. Die Repräsentationszonen werden dem Bewegungssystem, den Sinnesorganen und dem Zentralen Nervensystem zugeordnet. Die YPSILON-Punkte stellen ein Somatotop im östlichen Sinne dar. Hierüber können Störungen des Meridiansystems, der «inneren Organe» im Sinne der TCM und der zugehörigen Analogien der Funktionskreise behandelt werden.

\section{BASIS-Punkte}

Die BASIS-Punkte (Abb. 1) befinden sich frontal bilateral und noch einmal in spiegelbildlicher Darstellung okzipital. Die frontalen BASISPunkte sind in der Behandlung von
Schmerzerkrankungen häufig den okzipitalen überlegen. Bei entsprechenden Erkrankungen/Störungen werden die BASIS-Punkte empfindlich und können gezielt akupunktiert werden. Detektiert wird mit der Fingerkuppe oder dem Fingernagel. Nach Palpation der lokalen Druckdolenz, die manchmal auch als Verhärtung oder trophische Störung getastet werden kann, wird die Akupunkturnadel unter fixierendem Finger in die druckdolente Stelle eingestochen. Das schmerzhafte Areal kann direkt unter der Hautoberfläche, dem Periost aufgelagert oder auch im Bereich des Musculus temporalis in einer Tiefe von ca. $0,5-1 \mathrm{~cm}$ liegen. Die Nadel sollte ca. 20 min in situ belassen werden und der Patient sollte sich entspannen.

Besonders bei akuten Beschwerden zeigt die Akupunktur der BASISPunkte eine rasche oder sofortige Beschwerdelinderung. Je öfter behandelt wird, desto länger hält die Beschwerdereduktion an. Prinzipiell werden die YNSA-Punkte ipsilateral der Beschwerdesymptomatik und lediglich
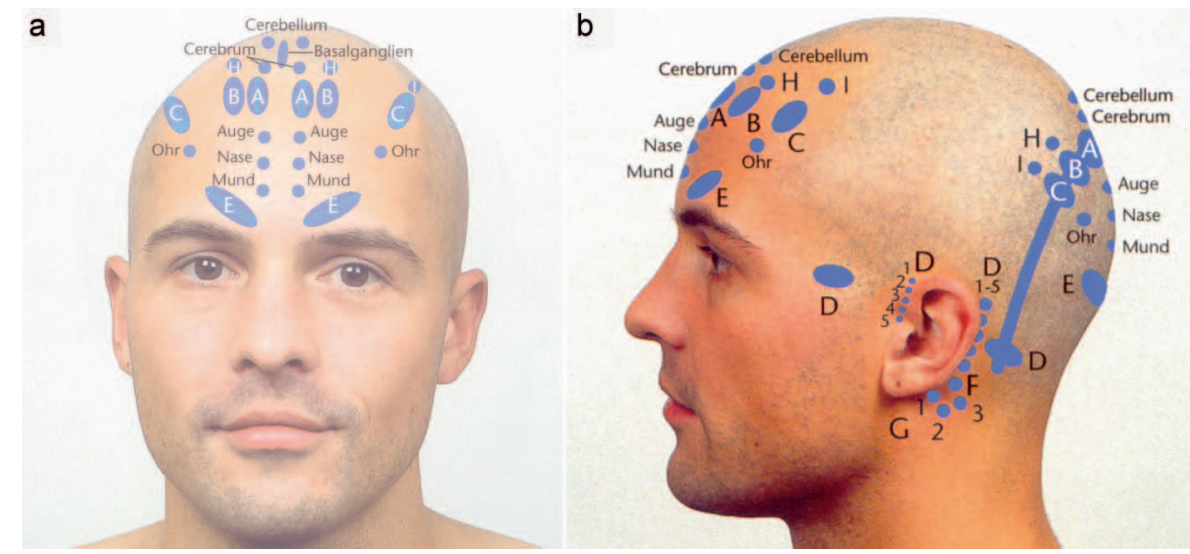

Abb. 1. YNSA-Basis-Punkte (mit freundlicher Genehmigung aus: Ogal HP, Kolster BC: Propädeutik der Neuen Schädelakupunktur nach Yamamoto (YNSA). Stuttgart, Hippokrates, 2004).

\section{KARGER}

Fax +497614520714 Information@Karger.de www.karger.com (c) 2012 S. Karger GmbH, Freiburg

Accessible online at: www.karger.com/szg
Dr. med. Hans P. Ogal

Leiter Schmerztherapie/Stellvertretender Chefarz

Aeskulap-Klinik

Gersauerstrasse 8, 6440 Brunnen, Schweiz

Tel. +41 41-8254747, Fax -8254782

hans.ogal@aeskulap.com 
bei zentral bedingten Lähmungen kontralateral eingesetzt.

Die repräsentierten Körperregionen werden in Tabelle 1 aufgeführt.

\section{YPSILON-Punkte}

Die frontalen YPSILON-Punkte (Abb. 2) befinden sich beidseits im Bereich der Schläfe. Sie liegen in einem Areal, das frontal von der idealen Schläfen-Haar-Grenze, kranial von einer gedachten horizontalen Begrenzung $2 \mathrm{~cm}$ kranial der Ohrspitze, okzipital vom vorderen Ohrmuschelansatz und kaudal vom Jochbogenoberrand begrenzt wird. Auch hier steht dem wichtigeren frontalen Somatotop ein okzipitales Somatotop gegenüber, das spiegelbildlich und leicht nach kaudal versetzt liegt. Bei Erkrankungen/Störungen der «inneren Organe» und ihrer Entspre- chungen, vor allem auch bei Schmerzsymptomatiken im Meridianverlauf, werden die korrespondierenden YPSILON-Punkte empfindlich und können gezielt akupunktiert werden. Somit ergeben sich die möglichen Indikationen der YPSILON-Punkte aus den Meridianverläufen und den Verbindungen der «inneren Organe der $\mathrm{TCM} »$ mit ihren analogen $\mathrm{Zu}$ ordnungen.

Die Auswahl, welcher YPSILONPunkt zur Behandlung indiziert ist, kann aufgrund seiner Schmerzempfindlichkeit bei der Palpation festgelegt werden. Es besteht aber weiterhin die Möglichkeit, anhand der Bauchdecken- und/oder Halsdiagnostik die Auswahl der indizierten Punkte zu treffen. Auch die diagnostischen Zonen der Bauchdecke und am Hals werden bei Schmerzsymptomatiken druckdolent und weisen den Weg zu den indizierten
YPSILON-Punkten. Die korrekte Akupunktur der YPSILON-Punkte lässt sich durch Kontrolle der Bauchdecken- und Halsdiagnostik (Abb. 3) überprüfen.

Eine Kombination von BASIS- und YPSILON-Punkten kann sich durchaus positiv ergänzen. Darüber hinaus kann die YNSA mit klassischer Akupunktur, aber auch mit allen anderen regulativen Verfahren kombiniert werden.

\section{Indikationen}

Alle funktionellen und prinzipiell reversiblen Störungen sowie Schmerzzustände des Bewegungssystems, zur Schmerztherapie und verbesserten Mobilisation nach Verletzungen und Operationen sowie zur Rehabilitation und Behandlung von zentralen und peripheren Lähmungen.

Tab. 1. Repräsentierte Körperregionen der BASIS-Punkte

\begin{tabular}{ll}
\hline A-Punkt & Kopf, Halswirbelsäule \\
B-Punkt & Halswirbelsäule, Nacken, Schulter \\
C-Punkt & Schulter, obere Extremität \\
D-Punkt & Lendenwirbelsäule, Becken, untere Extremität \\
Punkte D1-D5 & Lumbalsegmente L1-L5 \\
E-Punkt & Thorax, Brustwirbelsäule, Abdomen \\
F-Punkt & Nervus ischiadicus \\
Punkte G1-G3 & Kniegelenksregion \\
H-Punkt & Lendenwirbelsäule \\
I-Punkt & Lendenwirbelsäule; Versorgungsbereich des N. ischiadicus \\
Sinnesorganpunkte & Augen, Nase, Mund und Ohren \\
Cerebrum-Punkt & Grosshirn \\
Cerebellum-Punkt & Kleinhirn \\
Basalganglien-Punkt & Stammhirnregion (Basalganglien) \\
\hline
\end{tabular}

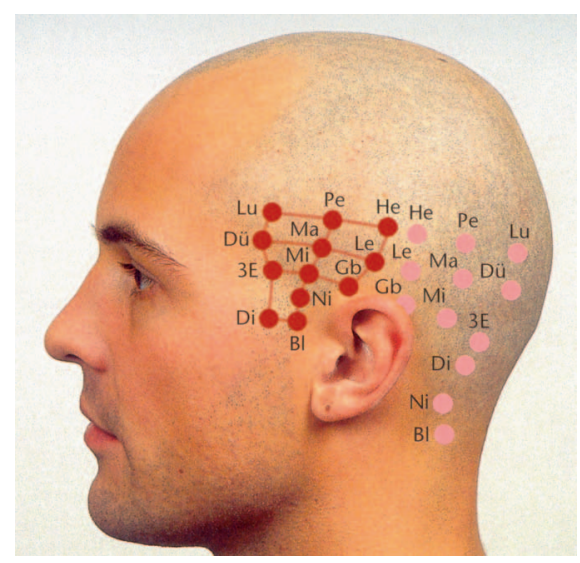

Abb. 2. YNSA-Ypsilon-Punkte (mit freundlicher Genehmigung aus: Ogal HP, Kolster BC: Propädeutik der Neuen Schädelaku-
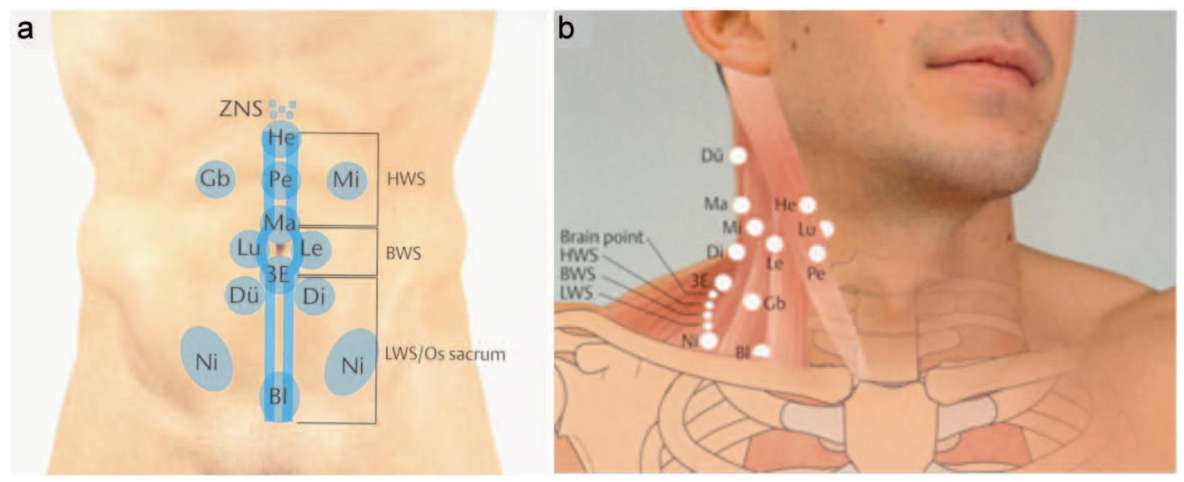
punktur nach Yamamoto (YNSA). Stuttgart, Hippokrates, 2004).

Abb. 3. a Bauchdeckendiagnostik. b Halsdiagnostik (mit freundlicher Genehmigung aus: Ogal HP, Kolster BC: Propädeutik der Neuen Schädelakupunktur nach Yamamoto (YNSA). Stuttgart, Hippokrates, 2004). 
Indikationsbeispiele für die ZNS-Punkte

Motorische Störungen, Hemiplegie, Paraplegie, Migräne, Morbus Parkinson, Vertigo, Sehstörungen, Tinnitus, Aphasie, Demenz, Insomnie, Morbus Alzheimer, Depressionen.

\section{Relative Kontraindikationen}

Unklare Schmerzzustände, fehlende Diagnose, extreme Erschöpfungs- und Schwächezustände, Vorsicht bei Schwangerschaft wegen Kollapsneigung, extreme Schmerzhaftigkeit einzelner Punkte.

\section{Kontraindikationen}

Lebensbedrohliche Krankheitsbilder, akute Schmerzen mit Operationsindikation, schwere Infektionserkrankungen, Entzündungen im Punktionsgebiet, akute Trigeminusneuralgie - ipsilaterale Akupunktur.

\section{Anstehende Kurse}

- 07. Juni 2012: Kongress der Österreichischen Wissenschaftlichen Ärztegesellschaft für Akupunktur (ÖWÄA); www.akupunktur.org

- 15.-17. Juni 2012: Hochschule Hannover, Arbeitsgemeinschaft Interdisziplinäre Medizin (AIM); www.aim-hannover.de

- 21./22. September 2012: ZÄN-Kongress Freudenstadt, Deutsche Ärztegesellschaft für Akupunktur (DÄGfA); www.daegfa.de

- 08.-12.10.2012 und 05.-09.11.2012: Miyazaki/Japan; Info: thcmkyet@ gmail.com

\section{Literatur}

Gleditsch JM: MAPS. MikroAkuPunktSysteme. Grundlagen und Praxis der somatotopischen Therapie. Stuttgart, Hippokrates, 2002.

Ogal HP: Neue Schädelakupunktur nach Yamamoto (YNSA); in Bäcker M, Hammes MG (Hrsg): Akupunktur in der Schmerztherapie. München, Urban und Fischer, 2005.

Ogal HP, Kolster BC: Propädeutik der Neuen Schädelakupunktur nach Yamamoto (YNSA). Stuttgart, Hippokrates, 2004.

Yamamoto T, Yamamoto H, Yamamoto MM: Yamamoto Neue Schädelakupunktur - YNSA. Kötzting, Verlag für Ganzheitliche Medizin, 2005.

Yamamoto T, Yamamoto H, Yamamoto MM: Yamamoto New Scalp Acupuncture YNSA. Miyazaki, Miyazaki Minami Printing Co., Ltd., 2010 . 